\title{
Reinforcing properties of conspicuous objects before imprinting has occurred
}

\author{
P. P. G. BATESON AND ELLEN P. REESE ${ }^{1}$ \\ SUB-DEPARTMENT OF ANIMAL BEHAVIOUR, MADINGLEY, CAMBRIDGE, ENGLAND
}

Day-old domestic chicks and Mallard ducklings rapidly learned to depress a pedal that switched on a flashing light. The flashing light, which the birds had never seen before, elicited filial behavior and was known to be highly effective as an imprinting object. Chicks that had been previously exposed to the light did not learn the problem any more quickly than those that had not.

Young birds that have not yet developed a specific social preference are sometimes thought of as waiting passively until they are exposed to a moving object, whereupon filial behavior is elicited and the process of imprinting begins. Some evidence suggests, however, that the birds search for objects that are conspicuous to the human eye before imprinting has occurred (Bateson, 1964; 1966). If conspicuous stimuli do, indeed, bring such behavior to an end and are consummatory, they might be expected to reinforce behavior of the young birds. It is already known that activities of previously imprinted birds can be reinforced by the presentation of the familiar object (Peterson, 1960; Campbell \& Pickleman, 1961; Hoffman et al, 1966). But it does not necessarily follow that all conspicuous stimuli are reinforcing from the outset; the familiar object might acquire such properties as the result of imprinting (Moltz, 1960; Bateson, 1966). Our concerm, therefore, was to determine whether the activities of young birds can be reinforced by conspicuous objects which they have never seen before.

The activity chosen for possible conditioning was stepping onto a pedal at floor level since it was easily performed by young chicks and ducklings and did not require shaping by us. Furthermore, it was not necessary to handle a bird once it had been placed in the apparatus.

\section{Method}

Fertile eggs of wild Mallard (Anas platyrhynchos) and domestic "Chunky" chicks (a broller strain) were obtained at least two days before they were due to hatch; the birds hatched in the dark. All 14 ducklings (Group A) and one group of seven chicks (Group B) were kept in a dark incubator until they were tested. Seven chicks in Group $C$ were isolated with food and water in llluminated cages 3-6 $\mathrm{h}$ after hatching. Seven chicks in Group $D$ were reared in the dark and imprinted with the flashing light, subsequently used as a possible reinforcer, 16-18 $\mathrm{h}$ after hatching and $6-8 \mathrm{~h}$ before testing.

The imprinting procedure for $\mathrm{Group} \mathrm{D}$ involved placing each chick singly at one end of an alley $120 \mathrm{~cm}$ in length with the flashing light behind a mesh screen at the other end. Having approached the light the chick was allowed $1 \mathrm{~min}$ in front of it, after which the light was turned off and an identical light turned on at the other end. In this way each chick travelled the whole length of the alley 10 times. After imprinting, each chick was returned to the dark incubator.

The operant conditioning apparatus is described in detail elsewhere (Bateson \& Reese, in press). The area of the apparatus over which the birds could move measured $37 \mathrm{~cm}$ wide $\times 15 \mathrm{~cm}$ deep. At the front corners two pedals, $7.5 \mathrm{~cm}$ square, were set into the floor. When a pedal was slightly depressed by the weight of a bird, a switch was closed. A flashing light was only turned on if the bird stepped onto one of the pedals; the other pedal served as a control, measuring any general changes in activity which might occur during a test. The $37 \mathrm{~cm}$ wide front of the apparatus was made of wire mesh. Centered in front of this screen and $20 \mathrm{~cm}$ away was the consplcuous stimulus. It consisted of a translucent plastic box (18 cm long, $9 \mathrm{~cm}$ wide, and $18 \mathrm{~cm}$ high) enclosing a 45-W 12-V bulb. When the pedal connected to it was pressed, the bulb lit up and the box rotated on a vertical axis at $85 \mathrm{rpm}$. The two smaller sides of the box were blacked out so the light appeared to flash. The flashing light remained on so long as the bird stayed on the pedal. The light was highly effective in eliciting approach responses and was positioned between the pedals so that a bird would have to come off the pedal in order to approach it. As soon as a bird did so the light went out.

Each bird was tested singly 22-25 $\mathrm{h}$ after hatching. If it had not learned to switch the light on within an hour of being placed in the apparatus, it was removed.

A bird was considered to have learned to switch on the light If, after coming off the pedal connected to the light, it tended to return to the pedal. Criterion was reached if it did so within 30 sec eight times out of 10 and if, during the 10 intervals between pressing the active pedal, it pressed the control pedal no more than twice. For purposes of comparison measurements were taken from the first occasion on which the active pedal was pressed to the first of the 10 responses in which criterion was reached.

\section{Results}

With the exception of one duckling and a chick in Group C, all the birds reached criterion. However, few birds learned to keep the light on continuously; as soon as it came on the majority tended to approach 


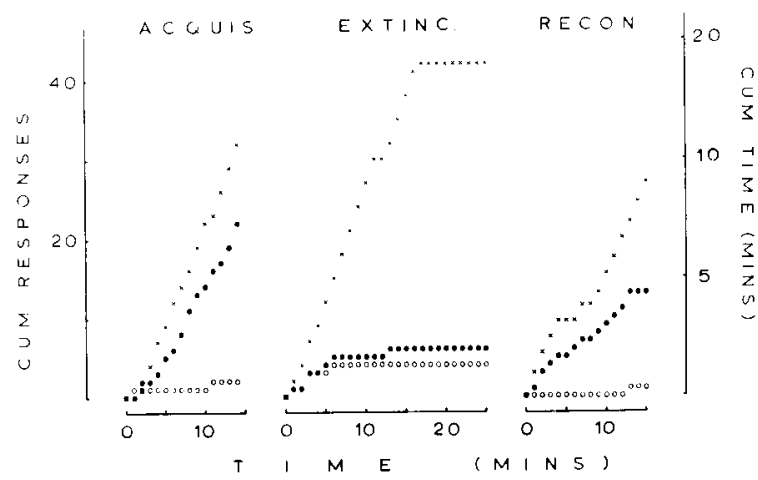

Fig. 1. Cumulative records of the number of presses of the reinforced pedal $(\bullet)$ and control pedal $(O)$, and the time spent on the reinforced pedal (X) obtained from a 24 h dark-reared domestic chick, during acquisition, extinction, and reconditioning. The reinforcer was a flashing light to which the chick has not been previously exposed.

it and as a consequence came off the pedal. For this reason, both the length of time on the active pedal and the number of presses of the active and control pedals were measured. Cumulative records of a $24 \mathrm{~h}$ dark-reared chick during acquisition, extinction, and reconditioning of the response are shown in Fig. 1. During acquisition, the chick repeatedly left the pedal while approaching the light and returned to the pedal when the light went out. During extinction, when the light was disconnected from the active pedal, the chick remained on this pedal for most of the first $16 \mathrm{~min}$ and left it only five times. Since the light was not operating the chick presumably had no cause to approach it. When the light was reconnected during reconditioning, the cumulative records of time spent on the active pedal and the number of times it was pressed increased once again, except for the final $2 \mathrm{~min}$ when the chick remained on the pedal.

The number of responses to reach the beginning of criterion is shown for each bird in Fig. 2. The imprinted chicks (Group D) took a significantly greater number of responses to reach criterion than did the darkreared chicks in Group B (Mann-Whitney $U=10, p<$ $0.05)$. None of the other differences between groups is statistically significant for this measure. The ducklings generally moved about more and trod on the control pedal significantly more often than the chicks (Mann-Whitney $\mathrm{U}=36, \mathrm{p}<0.01$ ), but no duckling or chick had pressed this pedal as often as the one connected to the light by the end of criterion.

\section{Discussion}

Day-old domestic chicks and Mallard ducklings learned in remarkably few responses to press a pedal which presented a conspicuous object. The object elicited filial behavior and was known from previous experiments to be highly effective as an imprinting stimulus. The rapidity with which the dark-reared birds, in particular, learned the problem suggests that they did not have to become familiar with the characteristics of the flashing light for it to have reinforcing properties. Indeed chicks that had previously been exposed to the

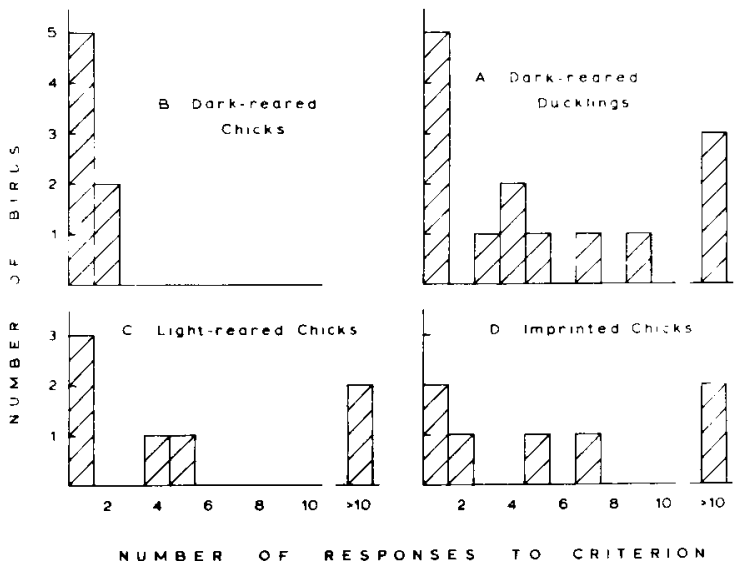

Fig. 2. The number of responses to criterion in each of four groups of day-old birds.

flashing light and presumably given ample opportunity to learn its characteristics did not behave differently from light-reared birds and took slightly longer to reach criterion than the dark-reared chicks. The effect of different rearing conditions is much more pronounced in older birds and is discussed elsewhere (Bateson \& Reese, in press).

It might be argued that any change in the visual modality would have reinforced the activities of the birds (cf. Kish, 1966), and that they would have worked to turn off the light as readily as they did to turn it on. However, this conclusion seems plausible only if the problem is divorced from its biological context, and we already have evidence that strongly links the reinforcing effects of light onset to the imprinting situation (Bateson \& Reese, in press).

Our results do not exclude the possiblilty that the reinforcing properties of the object to which a bird has been exposed may be further enhanced as a result of imprinting. They do, however, strongly suggest that before imprinting has occurred, the stimulation for which the birds seem to search can reinforce an arbitrarily chosen activity.

\section{References}

BATESON, P. P. G. Changes in the activity of isolated chicks over the first week after hatching. Anim. Behav., 1964, 12, 490-492.

BATESON, P. P. G. The characteristics and context of imprinting. Biol. Rev., 1966, 41, 177-220.

BATESON, P. P. G., \& REESE, E. P. The reinforcing properties of conspicuous stimuli in the imprinting situation. Anim. Behav., in press.

CAMPBELL, B. A., \& PICKLEMAN, J. R. The imprinting object as a reinforcing stimulus. J. comp. physiol. P\$chol, 1961, 54, 592-596.

HOFFMAN, H. S., SEARLE, J. L., TOFFEY, S., \& KOZMA, F., Jr. Behavioral control by an imprinted stimulus. J. exp. Anal. Behav., $1966,9,177-189$.

KISH, G. B. Studies of sensory reinforcement. In W. K. HONIG (Ed.), Operant behavior: areas of research and application. New York: Appleton-Century-Crofts, 1966.

MOLTZ, H. Imprinting: empirical basis and theoretical significance. Psychol. Bull., 1960, 57, 291-314.

PETERSON, N. Control of behavior by presentation of an imprinted stimulus. Science, 1960, 132, 1395-1396.

Note.

1. Now at Mount Holyoke College, South Hadley, Mass., 01075. 